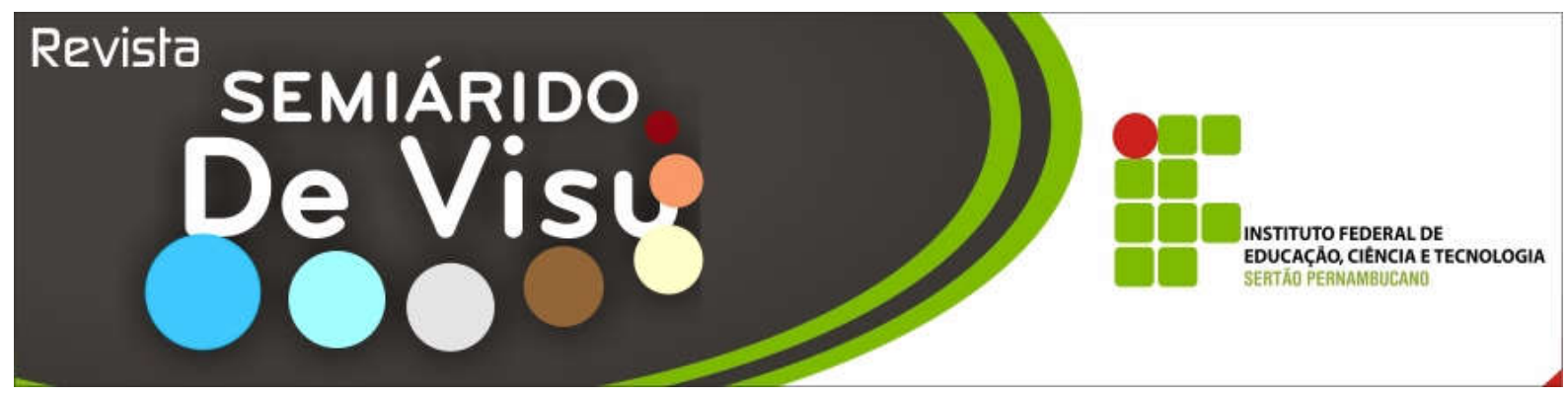

\title{
Ensino de Física: experimentação com analogia entre a eletrização do canudo e o circuito $\mathbf{R C}$
}

\author{
Thiago Alves de Sá Muniz Sampaioํㅜ, Cicero Jailton de Morais Souza², Eriverton da Silva Rodrigues ${ }^{3}$ \\ 1,2,3 Instituto Fe deral de Educ ação, Ciência e Tec no logia do Sertão Pernambuc ano. Campus de Salgueiro/PE. Rodovia Luiz Gonzag a
} (BR 232), KM 508, ZONA RURAL, CEP 56000-000, Tele fone: (87) 3421 -0050. E-mail: alveshere @, hotmail.com

RESUMO: Com base numa discussão histórica e de acordo com as propostas dos PCNs abordamos um experimento simples que possa envolver os alunos nos conteúdos iniciais da eletrostática e, além disso, que possa ser feita uma conexão de conc eitos entre movimentos de cargas e o circuito RC. O uso de analogias também continua servindo como metodologia para o ensino da Física, como por exemplo, para o ensino de circuitos elétricos, o ensino de Óptica e de eletricidade. Isso ressalta o quanto é importante se fazer comparações de fenômenos buscando uma melhor compreensão dos conceitos envolvidos no ensino-aprendizagem de Física. Após a execução deste trabalho esperamos fazer com que os alunos sejam motivados e incentivados, sendo estes os multiplicadores do conhecimento científico. Também temos a expectativa de que com uma nova forma de ensino-aprendizagem, associada a utilização de um experimento durante a execução do projeto, demonstre para todos o aproveitamento da Física Experimental para o ensino fundamental, médio e superior.

Pala vras-chave: Capacitores, Descarga, Ele tros tática.

\section{PHYSICAL EDUCATION: TRIAL WITH ANALOGY BETWEEN THE ELECTRIFICATION OF THE STRAWAND CIRCUIT RC}

\begin{abstract}
Based on a historical discussion and in accordance with the proposals of PCNs, we approach a simple experiment that can engage students in the initial contents of electrostatic and moreover, that there can be a connection between concepts of charges movement and the RC circuit. The use of analogies also continues to serve as a methodology for teaching physics, for example, for teaching electrical circuits, teaching optics and electricity. This underscores how important it is to make comparisons of phenomena seeking a better understanding of the concepts involved in the teaching of Physics. Upon execution of this project we hope to make the students are motivated and encouraged, which are multipliers of scientific knowledge. We also expect that with a new way of teaching and leaming, coupled with the use of an experiment during the execution of the project, show for everyone the utilization of experimental physics for elementary school, high school and college.
\end{abstract}

key words: Capac itors, Discharge, Electro static. 


\section{Introdução}

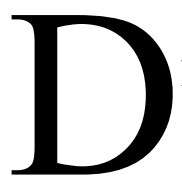
esde as épocas mais remotas da história da humanidade têm-se contribuições de filósofos, escritores, pesquisadores e cientistas para as atividades retribuírem em benefícios para a comunidade, iniciando com as explicações no que se refere à formação da matéria. As experiências de eletrização por atrito são conhecidas há mais de 2 mil anos (Tales de Mileto, 600 a.C.). Na Grécia antiga, sabia-se que o âmbar, uma resina (seiva de árvores solidificadas ao longo de séculos), quando atritado com pele de animais, atraía partículas leves, como sementes ou fragmentos de palhas. O nome do âmbar, em grego é "elektron": a este termo se atribui à origem da palavra eletricidade e do nome da partícula elementar elétron. (CHAVES, 2012).

O estudo sistemático da eletricidade tem seu início no século XVII com os trabalhos de estudiosos como Otto Guericke (1602-1686), Francis Hauksbee (1660-1713), Stephen Gray (1666-1736), Charles François de Cisternay Du Fay (1698-1739), William Gilbert (1544-1603), Benjamin Franklin (1706-1790), entre outros (SILVA, 2008). Em 1600, William Gilbert, médico da corte na Inglaterra, publicou seu tratado De mangnete, onde menciona outros corpos que se eletrizam por atrito, tais como: o vidro, o enxofre e o lacre (resina colorida) (ROONEY, 2013). No século XVII, a eletricidade também estudada por Gilbert era pouco mais que motivo para diversão. Benjamin Franklin (1706-1790), com interesse pela eletricidade, contribuiu intensamente com suas experiências, fornecendo bases para que Coulomb comprovasse, posteriormente, a lei eletrostática que rege as forças de atração e repulsão entre corpos eletrizados, por atrito, contato e indução (ROONEY, 2013) (GASPAR, 2003).

A partir do que foi tratado até agora, percebe-se a preocupação de muitos dos cientistas da época com a busca por analogias de conceitos envolvendo processos de eletrização e suas posteriores concepções, o que pode ser constatado pela bibliografia atual no ensino de ciências (BOZELLI e NARDI,
2006). Daí, o uso da experimentação é de suma importância em tal estudo, pois fez com que os cientistas pudessem compreender os fenômenos físicos de acordo com os pensamentos da época (GASPAR, 2003) (PINHO ALVES, 2000).

Muitos dos livros didáticos $\mathrm{e}$ conceituais da área (HEWITT, 2011) (HALLIDAY, 1995) (LUZ e ALVARES, 2005) não trazem esses tipos de analogias com enfoque em atividades experimentais na eletrostática. No entanto, esse tipo de abordagem é bastante eficaz para a chamada aprendizagem significativa dos alunos, visto que diferentes enfoques nas atividades experimentais com uso de analogias promove uma conexão de novos conhecimentos com os conhecimentos prévios dos alunos (ARAÚJO e ABIB, 2003).

Com base nessa discussão histórica e de acordo com as propostas dos PCN's (BRASIL, 2002) (SANTOS, 2002) abordamos um experimento simples que possa envolver os alunos nos conteúdos iniciais da eletrostática e, além disso, que possa ser feita uma conexão de conceitos entre movimentos de cargas e o circuito RC. O uso de analogias também continua servindo como metodologia para o ensino da Física, como por exemplo, para o ensino de circuitos elétricos, o ensino de Óptica e de Eletricidade. Isso ressalta o quanto é importante se fazer comparações de fenômenos buscando uma melhor compreensão dos conceitos envolvidos no ensino-aprendizagem de Física.

\section{Material e Métodos}

A metodologia desse trabalho é voltada para uma problematização de um conteúdo conectando os conhecimentos prévios dos alunos aos conceitos físicos envolvidos, fazendo com que os alunos interajam com o experimento desde o processo de construção até a apresentação, o que pode proporcionar a chamada aprendizagem significativa.

Os procedimentos foram realizados no período de Janeiro a Março de 2015, sempre das 13:00hs às 17:00hs, em ambiente fechado a 
uma temperatura média aproximada de $25^{\circ} \mathrm{C}$ (a medição da temperatura foi realizada com o sensor do Arduino). O processo se deu no laboratório de física do IF-Sertão PE Campus Salgueiro.

Para realização da primeira etapa do experimento foram usados canudos plásticos para sucos ou refrigerantes, papel tipo toalha, multímetro e um eletroscópio montado de maneira experimental. O eletroscópio consistia apenas de uma bateria de $9 \mathrm{v}$ conectada em série com o multímetro, com um resistor de $5 \mathrm{k} \Omega$ e com coletor e a base de um transistor BF-245, seu emissor é então conectado em serie com um resistor de $5 \mathrm{M} \Omega$ e com um fio de cobre de $25 \mathrm{~cm}$ de comprimento e $1 \mathrm{~mm}$ de espessura (que funciona como uma antena).
$\mathrm{O}$ experimento consistiu em prender a base dos canudos a uma distância fixa de $5 \mathrm{~cm}$ da antena do eletroscópio para, em seguida, atritá-los com um papel tipo toalha. Ao fazer isso, o canudo se carregava e o multímetro indicava a medida da diferença de potencial em função do tempo (Figura 1). Com o uso de uma webcam, filmamos o multímetro durante todos os processos. Com as filmagens, conseguimos tirar os dados para intervalos de tempo da ordem de um segundo, colocando-os em uma planilha eletrônica para, por fim, construirmos o gráfico da tensão em função do tempo. Este procedimento foi repetido diversas vezes com canudos de três marcas diferentes (feitos de polietileno e polipropileno).

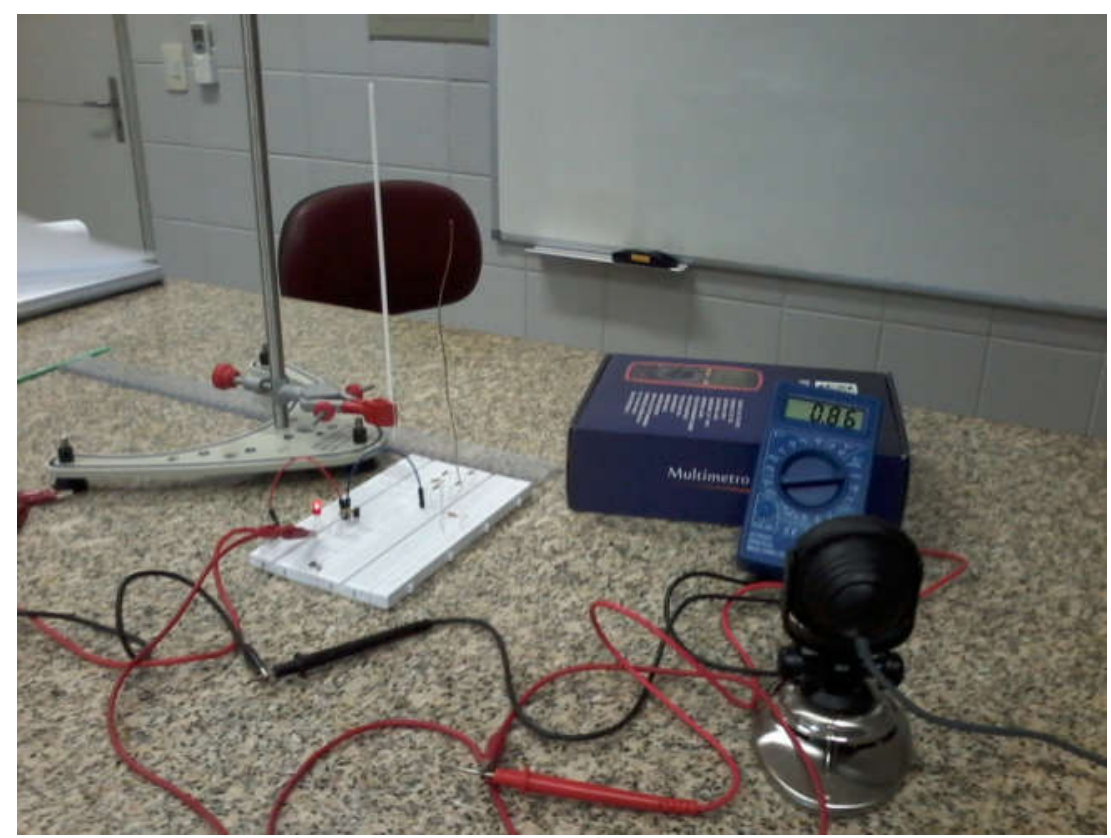

Figura 1. Experimento para obtenção dos dados. A antena captava a variação do campo eletrostático do canudo carregado e através do transistor transmitia os dados ao multímetro, enquanto ele era filmado com a webcam.

Em cada medida, inicialmente o canudo e o papel encontravam-se neutros, ou seja, com a mesma quantidade de cargas positivas e negativas. Ao atritarmos o canudo com o papel, o canudo adquiria uma carga negativa (de acordo com a série triboelétrica).

Durante o experimento deve-se ressaltar que os canudos e os papéis foram diferentes para cada medida (mas, de mesmo fabricante); a eletrização por atrito do papel com o canudo foi sempre na direção vertical, de baixo para cima e o posicionamento do canudo foi o mesmo em todas as medidas. Além disso, as medições foram feitas por uma única pessoa e os canudos foram da cor branca.

Já para a obtenção dos dados de descarga do capacitor, fizemos a montagem do circuito RC básico e, com o auxílio de um microprocessador chamado Arduino, iniciamos a contagem dos dados. O processo funcionou 
da seguinte maneira: o hardware era conectado diretamente ao circuito, coletando as informações que eram previamente programadas no software pelo computador, que através de um cabo USB realizava a conexão entre $o$ circuito $e \quad o$ PC. Inicialmente, programamos o Arduino para coletar dados de tensão em função do tempo, e para isto, montamos um circuito onde um capacitor de $100 \mu \mathrm{F}$ se carregava e descarregava em seguida em um resistor de $10 \mathrm{k} \Omega$. A contagem dos dados foi feita instantaneamente com intervalos de tempo da ordem de milissegundos, para em seguida colocarmos os dados na planilha eletrônica e obtermos o gráfico que desejávamos

Para confirmação da analogia entre a eletrização do canudo e o circuito $R C$, esboçamos os gráficos a partir dos dados obtidos, servindo de meios para comparação experimental. Além disso, foram feitas correlações entre os resultados obtidos para determinar as equações correspondentes ao processo de "descarga" da eletrização do canudo.Com os dados obtidos e uso de planilhas eletrônicas obteve-se uma linha de tendência da carga em função do tempo. $\mathrm{Ou}$ seja, os dados obtidos puderam ser comparados ao processo de um capacitor descarregando.

\section{Resultados e Discussão}

Sabendo que todos os corpos são constituídos de átomos, e todo átomo possui um núcleo onde se localizam os prótons, com carga positiva, e uma camada externa onde se movimentam os elétrons com carga negativa. Ao atritarmos o canudo e o papel, fornecemos energia aos seus átomos. Isso faz com que os seus elétrons movimentem-se de um corpo para outro. De acordo com o princípio da conservação da carga, um dos corpos adquire elétrons ficando carregado negativamente e o outro que perdeu elétrons fica carregado positivamente.

Para a situação de eletrização por atrito que envolve o canudo e o papel, tem-se que quando o canudo é carregado eletricamente, este tem a propriedade de atrair pequenos pedaços de papel. Também tem a propriedade de ficar preso à parede enquanto não houver passagem de cargas elétricas da parede para o canudo e vice-versa.

Quando se atritam dois corpos, ambos ficam em contato íntimo um com o outro, o que pode fazer com que os átomos da superfície de um deles cedam elétrons para o outro. É o que acontece basicamente no nosso experimento, o canudo se eletriza por atrito com o papel, este por sua vez repele as cargas iguais da antena, a região da antena próxima ao canudo passa a ter carga oposta. $\mathrm{O}$ excesso de carga negativa no canudo repele as cargas negativas da antena, de modo que na antena em se concentrem apenas cargas positivas (pois são atraídas). O fluxo de cargas negativas vai para o transistor alterando, assim, a corrente elétrica total que passa pelo multímetro. Com o passar do tempo, o atrito (ou contato) do canudo com o ar em volta vai redistribuindo as cargas em excesso do canudo através do ar para a Terra, até que o canudo fique completamente neutro.

Com a ajuda da planilha eletrônica, procurou-se uma linha de tendência que explicasse o fenômeno. Como este experimento foi realizado várias vezes (com diferentes canudos), percebemos que todos os gráficos assumiram essa mesma forma, portanto escolhemos um gráfico representativo para servir de comparação. O gráfico da figura 2 representa o processo de um canudo em "descarga" (consideramos descarga como a taxa de troca de cargas entre o canudo e o ar). 
(SAMPAIO; SOUZA, RODRIGUES, 2015)

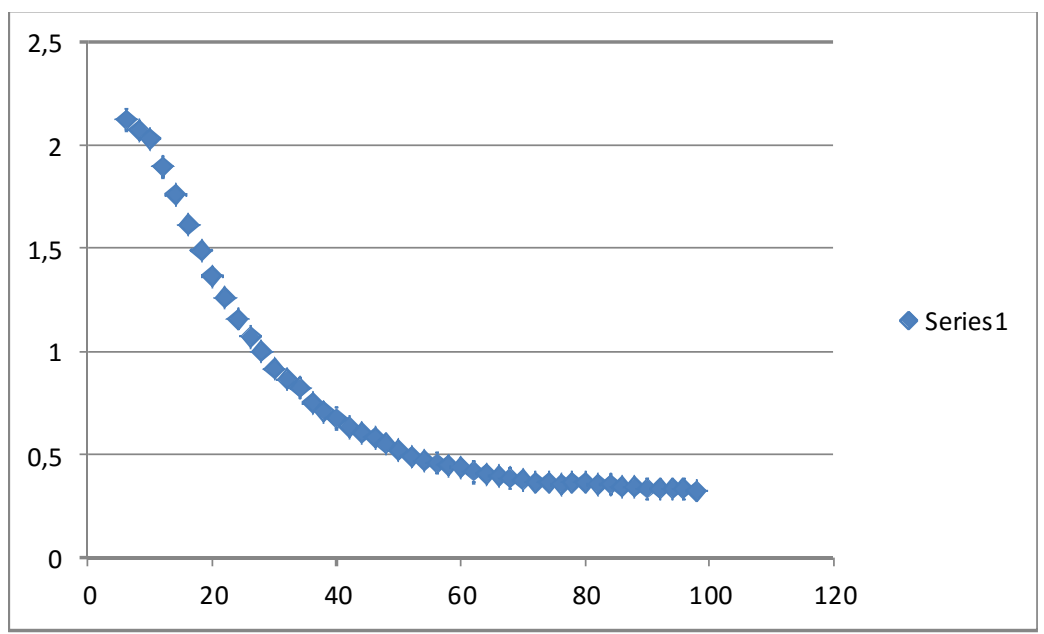

Figura 2. Tensão em função do tempo para o caso dos canudos.

$\mathrm{Na}$ figura 3, temos o gráfico obtido na situação de descarga do capacitor (com as medidas realizadas pelo Arduino) podemos ver o caráter exponencial previsto pela teoria.

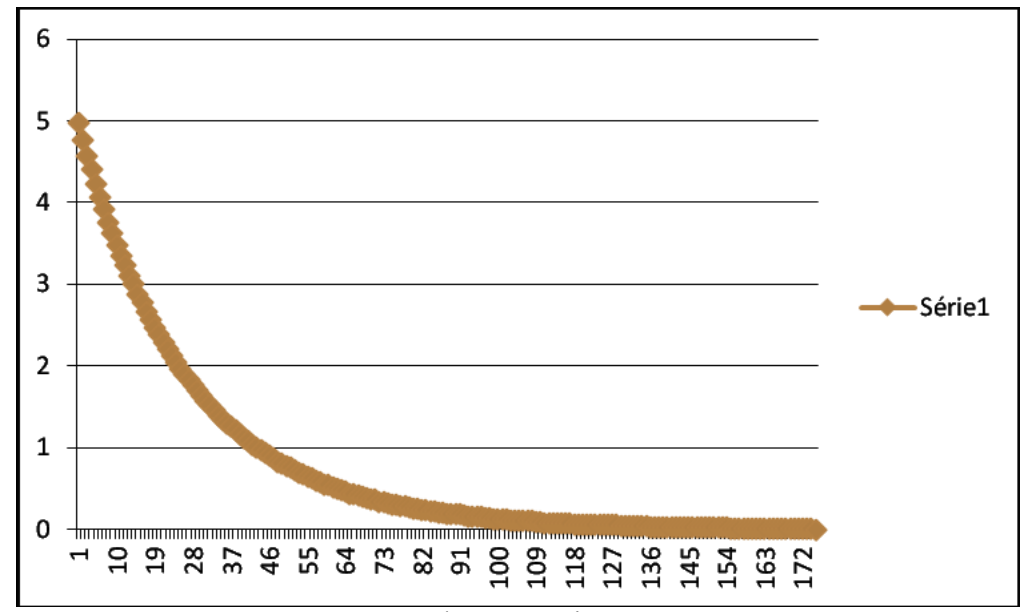

Figura 3. Descarga do capacitor - Tensão x Tempo

Pela equação a seguir de descarga do capacitor, temos que:

$$
\mathrm{V}=\mathrm{Vo} \quad \mathrm{e}^{-\mathrm{t} / \tau}
$$

Da equação 1, Vo é a voltagem inicial com o capacitor carregado, $\mathrm{V}$ a voltagem final (no caso tende a zero), t o tempo total de descarga, e $\tau$ (tau) é a constante de tempo do capacitor utilizado, que corresponde ao tempo para que um capacitor se descarregue até $63,2 \%$ do valor da sua carga total (ou da tensão, como são proporcionais).

Com o uso da planilha eletrônica, construímos a equação que representava $o$ gráfico da figura 2, que segue abaixo:

$$
\mathrm{V}=\mathrm{A} \quad \mathrm{e}^{-\mathrm{t} / \mathrm{B}}+\mathrm{C}
$$

Onde A, B e C são constantes. Como o experimento dos canudos foi repetido diversas vezes, os valores dessas constantes calculadas pelo programa mudaram em cada processo. Estes valores dependem fortemente das condições de contorno do processo. Uma variação ínfima na quantidade de cargas e nas condições do ambiente pode alterar sensivelmente as constantes. Porém a análise das constantes foge aos objetivos deste trabalho, que se limita apenas a mostrar o caráter exponencial dos processos. 
Por fim, comparando os gráficos da figura 3 e da figura 2, podemos inferir que o processo de "descarga" dos canudos apresenta uma queda exponencial em função do tempo. Esse caso é perfeitamente similar ao da descarga de um capacitor conectado a um circuito RC de tensão contínua. Vemos pelas equações 1 e 2 que os processos são bastante parecidos no que diz respeito ao seu caráter exponencial.

\section{Conclusões}

Esse trabalho estudou experimentalmente o fenômeno de eletrização de canudos, tecendo uma analogia entre o processo de descarga do canudo eletrizado com a descarga de um capacitor em um circuito RC. Essa abordagem simples pode permitir uma problematização do con teúdo, fazendo com que os alunos possam interagir com o experimento desde sua execução até a apresentação, permitindo uma compreensão dos fenômenos físicos. Ou melhor, verificar como se dá uma ideia da marcha em busca de um "modelo matemático" para um fenômeno físico. Além disso, conclui-se que o comportamen to de troca de cargas de um canudo com outro meio qualquer tem uma semelhança bastante evidente com o fenômeno de descarga de um capacitor.

\section{Referências}

ARAÚJO, Mauro S. T.; ABIB, Maria L. V. S. Atividades Experimentais no Ensino de Física: diferentes enfoques, diferentes finalidades. Revista Brasileira de Ensino de Física, São Paulo, v.25, n.2, p. 176-194, jun. 2003.

CHAVES, Alaor. Física bá sica. Rio de Janeiro: v. 3, LTC, 2012.

GASPAR, Alberto. Experiências de Ciências; São Paulo, Editora Ática; 2003 (1ª edição).

GASPAR, Alberto. Física - Eletromagnetismo e Física Moderna; São Paulo, Editora Ática; 2003 ( $1^{\text {a }}$ ediç̧ão).
BRASIL, PCN+ Ensino Médio. Orientações Educacionais Complementares aos Parâmetros Curriculares Nacionais: Ciências da Natureza, Matemática e suas Tecnologias (MEC-SEMTEC, Brasília, 2002).

HALLIDAY, David; RESNICK, Robert; WALKER, Jearl. Fundamentos de Física 3, 4a. Ed ição. Editora LTC. Rio de Janeiro, 1995.

HEWITT, Paul G. Física Conceitual; Tradução: Trieste Freire Ricci; revisão técnica: Maria Helena Gravina. - 11. Ed.- Porto Alegre; Bookman, 2011.

LUZ, A. M. R. e Alvares, B. A. Curso de Física - Volume 3. São Paulo: Scipione, 2005.

PINHO ALVES, J. Regras da transposição didática aplicadas ao laboratório didático.Caderno Catarinense de Ensino de Física, Florianópolis, v. 17, n. 2, p. 174-188, ag. 2000.

BOZELLI, Fernanda Kátia; NARDI, Roberto. $\mathrm{O}$ uso de analogias no ensino de Física em nível universitário: Interpretações sobre os discursos do professor e dos alunos. Revista Brasileira de Pesquisa em Educação em Ciências, v. 6, n. 3, p. 77-100, 2006.

ROONEY, Anne. A História da Física - da Filosofia ao Enigma da Matéria Negra; São Paulo, Editora M. Books; 2013 ( $1^{\text {a }}$ edição).

SANTOS, Lucíola Lucínio. Políticas Públicas para o ensino fundamental: Parâmetros Curriculares Nacionais e Sistema Nacional de Avaliação. In: Revista Educação e Sociedade. São Paulo: Cortez; Campinas: Cedes, V.23, n. 80, set. 2002.

SILVA, Cibele Celestino; PIMENTEL, Ana Carolina. Uma análise da história da eletricidade pres ente em livros didáticos: o caso de Benjamin Franklin. Caderno Brasileiro de Ensino de Física, v. 25, n. 1, p. 141-159, 2008. 